\title{
f-MAC: A Deterministic Media Access Control Protocol Without Time Synchronization
}

\author{
Utz Roedig, Andre Barroso and Cormac J. Sreenan \\ Mobile and Internet Systems Laboratory (MISL), \\ Computer Science Department, University College Cork (UCC), Ireland \\ $\{$ u.roedig, a.barroso, c.sreenan\}@cs.ucc.ie
}

\begin{abstract}
Nodes in a wireless network transmit messages through a shared medium. Thus, a Media Access Control (MAC) protocol is necessary to regulate and coordinate medium access. For some application areas it is necessary to have a deterministic MAC protocol which can give guarantees on message delay and channel throughput. Schedule based MAC protocols, based on time synchronization among nodes, are currently used to implement deterministic MAC protocols. Time synchronization is difficult and costly, especially in energy constrained sensor networks. In this paper the $\mathrm{f}-\mathrm{MAC}$ protocol is presented which can give guarantees regarding message delay and channel throughput without the requirement of time synchronization among nodes. The various trade-offs of f-MAC are analysed and discussed and application areas that would benefit from f-MAC are presented.
\end{abstract}

\section{Introduction}

Nodes in a wireless network transmit messages through a shared medium. Thus, some form of organization among the nodes is necessary to enable an effective usage of the shared resource. This organization is implemented by a Media Access Control (MAC) protocol that each node has to obey. Currently a number of varying MAC protocols with different properties and requirements exist. These properties might be high data throughput or transmission delay guarantees. Requirements might be time synchronization among all nodes or the capability of detecting a busy channel (carrier sense).

Most MAC protocols used in wireless networks today can be divided into two major classes: contention and schedule based MAC protocols. Both types differ in their properties and requirements.

A contention based MAC protocol is relatively simple to implement since there is no coordination necessary among the nodes. A node simply detects if the channel is currently used by another node; if not, the message is transmitted. If the channel is busy, the node backs off and tries again after some time. The problem of such a MAC protocol is that no worst-case guarantees can be given regarding throughput or maximum transmission delay. However, many application areas, for example medical applications, require strict guarantees. 
A schedule based MAC protocol is more difficult to implement because accurate time synchronization among neighbouring nodes is required. Each node uses a dedicated time slot to transmit messages. As fixed time slots are used, guarantees regarding bandwidth and message delay can be given. The main problem of such a MAC protocol is the complexity introduced by time synchronization. Especially in highly constrained sensor networks the synchronization overhead might not be acceptable.

This paper presents the $f-M A C$ protocol which overcomes the aforementioned restrictions. The protocol has, among other benefits shown in the paper, the following main features:

1. Bandwidth and delay guarantees are provided.

2. Time synchronization among nodes is not necessary.

The f-MAC protocol uses a framelet approach: fixed sized frames are retransmitted a fixed number of times with a specific frequency. Thus, the abbreviation f-MAC is used to refer to the presented protocol. As it will be shown in the paper, the capability of giving guarantees is traded for a lower bandwidth and higher transmission delay. However, for many application areas this is acceptable as hard guarantees are considered to be the most important design goal.

The remaining paper is organized as follows. In Section 2, the functionality and requirements of f-MAC are described. In Section 3 basic properties such as bandwidth and transmission delay are investigated analytically and by experiment. Section 4 compares the delivery probability of a simple random MAC with f-MAC. Section 5 describes cluster forming issues in larger f-MAC based networks. Section 6 shows application areas that benefit from the use of f-MAC. In Section 7 related work is discussed. Section 8 concludes the paper.

\section{2 f-MAC Concept}

In this section, the basic concept of f-MAC is presented. f-MAC uses a framelet approach as it is described in detail in [1].

\subsection{The Framelet Approach}

In the framelet approach, the same message is transmitted several times using small, fixed sized data packets. Each data packet of the transmission is called a framelet. A message transmission via the framelet approach is depicted in Fig. 1. The transmission duration of a framelet is denoted as $d$. Each transmission consists of $r$ framelets and the framelets are sent with a frequency of $f=1 / t$.

Certain types of ultra low-power transceivers, such as the Nordic nRF2401 [3], the Chipcon CC2420 [5] or the nanoNET TRX [4], are able to transmit small, fixed sized packets at a high speeds of typically $1 \mathrm{Mb} / \mathrm{s}$. These transceiver types are currently used in sensor networks and are capable of supporting the framelet approach. 
A framelet approach is normally used to increase transmission reliability or to allow a power efficient operation of the transceivers (see [1,2], implementation of duty cycles). As the same information is transmitted several times, the available bandwidth is reduced. However, in many cases reliability and especially power efficiency is considered more important than a high throughput.

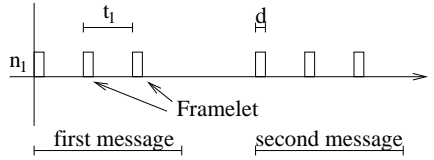

Fig. 1. Framelet transmission.

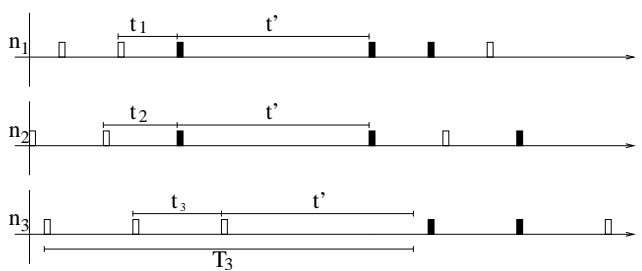

Fig. 2. f-MAC operation.

\subsection{Collision handling using Framelets}

Instead of using the framelet approach to increase transmission reliability or to allow power efficient transceiver operation, it can be used to deal with the problem of collisions. If several nodes in the same radio range transmit data via a set of framelets, collisions can still occur. However, if each node uses a specific unique framelet transmission frequency $f_{i}$, it is possible to ensure that one framelet of a set is always received, even if collisions are not prevented. This is the basic idea behind the f-MAC protocol and is explained in detail in the next paragraph.

\subsection{Framelet Media Access Control}

In f-MAC, no collision detection or time synchronisation between the nodes is used. The number of framelets per message and the framelet frequencies are selected such that it is guaranteed that at least one framelet per message is delivered without collision. f-MAC defines the following simple transmission policy:

Rule\#1 Each node has to transmit messages as framelets. The framelet length $d$ is defined by the f-MAC base unit $\delta$ as follows:

$$
d=\delta / 2
$$

Rule\#2 The number of framelets $r$ per message is defined by the number of nodes $N$ in transmission range.

$$
r=N
$$


Rule\#3 Each node $n_{i}$ has to use a specific framelet frequency $f_{i}=1 / t_{i}=$ $1 /\left(k_{i} \cdot \delta\right)$. The $k_{i} \in N^{+}$must satisfy the following equation:

$$
k_{i} \cdot(r-1)<\operatorname{LCM}\left(k_{i}, k_{j}\right) \quad \forall k_{i}<k_{j} 1 \leq i, j \leq N
$$

Rule\#4 After the start of the transmission of the last framelet of a message, a node must wait at least the time $t^{\prime}$ before a next message can be transmitted. $t^{\prime}$ is computed as:

$$
\begin{gathered}
k_{\max }=\max _{0 \leq i \leq N}\left\{k_{i}\right\} \\
t^{\prime}=\left(k_{\max } \cdot(r-1)+1\right) \cdot \delta
\end{gathered}
$$

From the previous described rules it can be deduced that a node $n_{i}$ needs in the worst case the following time $T_{i}$ to transmit a message:

$$
T_{i}=(r-1) \cdot t_{i}+t^{\prime}
$$

Example Fig. 2 shows an example with three nodes $(N=3)$. The number of framelets is therefore $r=3$. A possible ${ }^{1}$ set of $k_{i}$ satisfying Equation (3) is $k_{1}=4, k_{2}=5$ and $k_{3}=6$. According to Equation (4), the nodes use $t^{\prime}=13 \cdot \delta$. In the example it is assumed that $n_{2}$ starts first at an arbitrary time with a message transmission. Shortly after, $n_{3}$ and then $n_{1}$ start a transmission as well. In the example, framelet\#3 of $n_{1}$ and $n_{2}$ collide. However, all other remaining framelets of each node's ongoing transmissions can be transmitted successfully. After the time $t^{\prime}$ of node $n_{1}$ and $n_{2}$ expires, both nodes start immediately a new transmission. Here framelet\# 1 of $n_{1}$ and $n_{2}$ collide and are destroyed. However a framelet of each transmission can be subsequently submitted successfully. $n_{3}$ sends a message some time after its waiting period $t^{\prime}$ is over. Framelet\#1 of this transmission collides with framelet\#2 of the ongoing transmission of node $n_{1}$. Framelet\#2 of the transmission of $n_{3}$ collides with the framelet\#3 of $n_{2}$. However the last framelet of $n_{3}$ 's transmission is successfully transmitted.

\subsection{Validation}

The previous stated example shows that the transmission scheme is feasible under certain conditions. However, it has to be shown that the transmission scheme is successful for all possible time shifts between the nodes.

Lemma 1. If node $n_{i}$ sends $u\left(u \in N^{+}\right)$consecutive messages $m_{i, u}$ and node $n_{j}$ sends $v\left(v \in N^{+}\right)$consecutive messages $m_{j, v}$, only framelets of exactly one message $m_{i, u}$ can collide with framelets of exactly one message $m_{j, v}$.

\footnotetext{
$\overline{1}^{1}$ This set of $k_{i}$ is possible, but not optimal. The optimal set of $k_{i}$ is shown in Section 3.
} 
Proof. Assume for the sake of contradiction that framelets of message $m_{i, u-1}$ and $m_{i, u}$ with $u \neq 1$ collide with message $m_{j, v}$. For this to happen, the following equation must be fulfilled (see Equation 5):

$$
t^{\prime}<(r-1) \cdot t_{j}+\delta
$$

The origin of this inequality can be described using the example shown in Fig. 2. The inequality describes the case where a message transmission of $n_{2}$ does fit between two consecutive transmissions of $n_{1}$. This inequality can be simpified the following way:

$$
\left(k_{\max } \cdot(r-1)+1\right) \cdot \delta<\left(k_{j} \cdot(r-1)+1\right) \cdot \delta \Rightarrow k_{\max }<k_{j}
$$

According to f-MAC Rule\#4 and Equation 4, this statement can not be fulfilled and thus contradicts the initial assumption.

Lemma 2. If node $n_{i}$ and node $n_{j}$ transmit a message, no more than one framelet of these two message transmissions can collide.

Proof. Assume that more than one framelet of the transmission collide. Therefore, after one collision happened, $n_{i}$ and $n_{j}$ must send again a framelet at the same time. This can only be achieved if $k_{i}$ and $k_{j}$ have a least common multiplier within the period $k_{i}$ is sending its framelets. This can be expressed as follows:

$$
\operatorname{LCM}\left(k_{i}, k_{j}\right)<k_{i} \cdot(r-1) \quad \forall k_{i}<k_{j} 1 \leq i, j \leq N
$$

This statement obviously contradicts Rule\#3 and Equation 3 and thus contradicts the initial assumption.

Theorem 1. At least one framelet of any message transmission will be transmitted collision-free ( $f$-MAC theorem).

Proof. According to Lemma 1 and 2 only one framelet collision per message transmission between two nodes can occur. According to f-MAC Rule\#2 and Equation 2 exactly $r=N$ framelets are transmitted. Thus, even under worst case conditions, only $N-1$ framelets can be affected by a collision as a node cannot produce a collision with itself.

\section{3 f-MAC Properties}

Two important properties of a MAC protocol are the message delay and the available bandwidth. For most MAC protocols, the figures of these properties vary with the number of nodes that participate in a collision domain. This is observed as well within f-MAC and analyzed within this section.

Regarding message delay it has to be noted that an upper bound can be computed. This is the main feature and design goal of f-MAC. Many available MAC protocols do not allow us to compute such a deterministic bound. For example collisions might occur and random back-off times are used. 
Regarding bandwidth it has to be pointed out that f-MAC's design goal is not an optimisation of the channel utilisation. In fact, the channel utilisation is reduced to enable strict guarantees for the message delay. Therefore, f-MAC has a naturally poor channel utilisation.

\subsection{Worst Case Message Delay}

The upper bound for the message delay between two nodes is given by the time $T_{\max } . T_{\max }$ is computed the following way using Equation 5:

$$
T_{\max }=\max _{0 \leq i \leq N}\left\{T_{i}\right\}
$$

f-MAC ensures that after a delay of $T_{\max }$ a message is delivered between two arbitrary nodes. This is the worst-case delay bound for all nodes. If just one specific node is investigated, it might be possible to give a better upper bound of the message delay. This is possible as some nodes are able to transmit messages faster than others (They have a $T_{i}<T_{\max }$ ). Thus, the worst case delay bound $T_{\min }$ for the fastest node of the set is defined as:

$$
T_{\text {min }}=\min _{0 \leq i \leq N}\left\{T_{i}\right\}
$$

To analyse how the times $T_{\min }$ and $T_{\max }$ increase with the number of nodes, a program was implemented to determine the combination of $k_{i}$ for a given $N$ that leads to a minimal $T_{\max }$. The result of this computation is shown in Table 1 and $T_{\min }$ and $T_{\max }$ are shown graphically in Fig. 3 (The value $T_{a v}$ is explained and obtained by simulation in Section 3.3).

Table 1. Sets satisfying the f-MAC condition.

\begin{tabular}{|c|c|c|c|c|}
\hline$N$ & Set $k_{i}$ & $T_{\min }$ & $T_{\max }$ & $T_{a v}$ \\
\hline \hline 2 & $\{2,3\}$ & $6 \delta$ & $7 \delta$ & $3 \delta$ \\
\hline 3 & $\{2,3,5\}$ & $15 \delta$ & $21 \delta$ & $8 \delta$ \\
\hline 4 & $\{3,4,5,7\}$ & $31 \delta$ & $43 \delta$ & $16 \delta$ \\
\hline 5 & $\{3,5,7,8,11\}$ & $57 \delta$ & $89 \delta$ & $32 \delta$ \\
\hline 6 & $\{5,7,8,9,11,13\}$ & $91 \delta$ & $131 \delta$ & $50 \delta$ \\
\hline 7 & $\{5,7,8,9,11,13,17\}$ & $133 \delta$ & $205 \delta$ & $70 \delta$ \\
\hline 8 & $\{5,9,11,13,14,16,17,19\}$ & $169 \delta$ & $267 \delta$ & $101 \delta$ \\
\hline
\end{tabular}

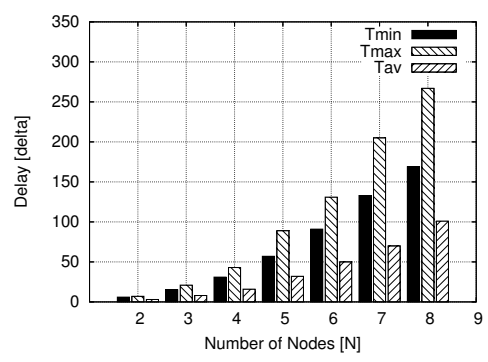

Fig. 3. Transmission delay $T_{\min }$ and $T_{\max }$ and $T_{a v}$.

As shown, the worst case delay bound increases exponentially with the number of nodes that are in the same collision domain. Obviously, the number of nodes used in one collision domain has a dramatic impact on the transfer delay. Thus, f-MAC might not be useful in highly populated networks. 
It also has to be noted that the gap between $T_{\min }$ and $T_{\max }$ increases with the number of nodes. Some nodes are able to send messages faster than others. This feature might be useful in cases where specific nodes have to deal with a high traffic load.

Example If, for example, $N=5$ nodes are selected and a Nordic nRF2401 transceiver [3] with a message size of $b=32$ bytes and a transmission speed of $1 \mathrm{Mbit} / \mathrm{s}$ is assumed, the following value for the transfer delay bound is obtained:

$$
\delta=2 \cdot 0.25 \mathrm{~ms}=0.5 \mathrm{~ms} \Rightarrow T_{\max }=89 \cdot 0.5 \mathrm{~ms}=44.5 \mathrm{~ms}
$$

\subsection{Bandwidth}

The available bandwidth $B_{i}$ between two arbitrary nodes is given by the time $T_{i}$ and the fixed framelet size $b$ in byte.

$$
B_{i}=\frac{b}{T_{i}}
$$

The available maximum bandwidth $B_{\max }$ (at the node using the smallest $k_{i}$ ) and minimal available bandwidth $B_{\min }$ can thus be calculated using $T_{\text {min }}$ and $T_{\max }$. The available bandwidth decreases exponentially as it depends on the exponentially increasing message delay bound $T_{i}$.

Example If, for example, $N=5$ nodes are selected and a Nordic nRF2401 transceiver [3] with a message size of $b=32$ bytes and a transmission speed of $1 \mathrm{Mbit} / \mathrm{s}$ is assumed, the following value is obtained:

$$
B_{\min }=\frac{32 \text { byte }}{44.5 \mathrm{~ms}}=7.2 \frac{\mathrm{kbit}}{\mathrm{s}}
$$

In this example, the maximum bandwidth is available for the node with $k_{i}=3$. In this case $T_{\min }$ is:

$$
T_{\min }=(r-1) \cdot t_{i}+t_{i}^{\prime}=4 \cdot 03 \cdot \delta+45 \cdot \delta=57 \cdot \delta \Rightarrow B_{\max }=11.23 \frac{\mathrm{kbit}}{\mathrm{s}}
$$

This small example shows that some nodes have a $T_{i}<T_{\max }$ and therefore have a higher bandwidth available.

\subsection{Average Message Delay}

As previously described, f-MAC guarantees that a message is delivered within the time $T_{\max }$ between arbitrary nodes. However, as each message transmission consists of several framelets, it is likely that the destination node will receive a message earlier than $T_{\max }$. In the previous paragraphs, the worst case upper 
bound for the message transfer delay was calculated. In this section, the average message transfer delay $T_{a v}$ between nodes is determined by simulation for specific traffic patterns.

If a node wants to transmit a message using the f-MAC protocol, three factors contribute to the observed message delay. First, the node $n_{i}$ might have to wait for a portion of $t^{\prime}$ before it is allowed to start the transmission of the framelets containing the message (see Section 2.3, f-MAC rules). Second, it depends which framelet of the framelet trail sent the receiver gets first. Third, the transfer delay depends on the $k_{i}$ of the f-MAC set the node is using to transmit. A node with a small $k_{i}$ repeats the framelets relatively fast and thus the receiver has a high chance to catch an uncollided framelet early.

One can expect that the average message transfer delay $T_{a v}$ is less than the guaranteed message delivery time $T_{\max }$. Obviously, the resulting average message transfer delay depends on the network configuration used and the particular traffic pattern.

Experiment The f-MAC protocol was implemented within a simulation environment. The ns2 network simulator was used for the experimental evaluation.

For the experiment, $N$ nodes $(2 \leq N \leq 8)$ are setup to transmit to one base station (star topology). All $N$ nodes are in the communication range of each other and the base-station.

For the experiment, a Nordic nRF2401 transceiver [3] with a message size of $b=32$ bytes and a transmission speed of $1 \mathrm{Mbit} / \mathrm{s}$ is assumed. Thus $\delta=0.5 \mathrm{~ms}$ is used. Each of the $N$ nodes creates new messages according to a Poisson distribution. The arrival rate $\lambda$ is the same at each node. Throughout all experiments, an arrival rate of a quater of the maximum possible rate, determined by the minimum available bandwidth $B_{\min }$ is used.

The experimentally obtained values of $T_{a v}$ are shown together with the analytically obtained results of $T_{\min }$ and $T_{\max }$ (see Section 3.1) in Table 1 and Fig. 3. As expected, the experiments show that the average message transfer delay is considerably better than the guaranteed maximum message transfer delay. If the arrival rate $\lambda$ is increased, the transfer delay $T_{a v}$ increases as more single framelet collisions occur. As expected, $T_{a v}$ is always - regardless of the $\lambda$ selected - smaller than $T_{\max }$.

Furthermore, the experiments show that the communication among all nodes can take place without any collision. The experiments show as well that the fMAC protocol implementation is relatively simple. To complete a full f-MAC implementation, only 100 lines of $\mathrm{C}++$ code were necessary. This simplicity makes the MAC protocol useful for implementation in the very constrained sensor network environment.

\subsection{Findings}

The guaranteed upper bound for the message delay and the available bandwidth depend heavily on the node density in the network. More specificly: 
1. The available bandwidth drops exponentially with the number of nodes.

2. The worst case delay increases exponentially with the number of nodes.

Therefore, it can be concluded that f-MAC is beneficial in sparsely populated networks where each node has only a few neighbours.

However, the experiments show that in realistic traffic scenarios, the worst case is not necessarily equal to the average operational case. Therefore the average message delay $T_{a v}$ is significantly smaller than the worst case message delay $T_{\max }$.

\section{Delivery Probability Analysis}

f-MAC provides $100 \%$ message delivery if it is assumed that messages are only lost due to collisions. In this section, the collision probability of a simple random transmission scheme is investigated. It is analysed how close such a simple MAC protocol will get to the design goal of $100 \%$ collision-free message delivery.

Under real world conditions, even a collision-free protocol can not guarantee $100 \%$ message delivery as the wireless channel is lossy ${ }^{2}$. Thus, one could argue that a MAC protocol does not need to be $100 \%$ collision-free; it would be enough if the probability of a message loss is not significantly increased by an additional collision probability. It is therefore of interest to know how a more primitive $\mathrm{MAC}$ protocol with properties similar to f-MAC performs regarding its collision probability. The comparison allows us to assess the operation conditions that justify the use of f-MAC instead of a more simple solution.

\subsection{Random Transmission Scheme}

The MAC protocol used for comparison with f-MAC is similar to the MAC protocol described and analysed in detail in [13].

The protocol is selected because it uses a framelet approach comparable to f-MAC. Different to f-MAC, the transmitted $r$ framelets are distributed in a random fashion within the time interval $T_{R S}$. The time interval $T_{R S}$ is divided in $r$ subintervals. A framelet of the current message is submitted within each subinterval. The transmission time of the framelet in the subinterval is given by a uniformly distributed random variable on $\left[0,\left(T_{R S} / r\right)-\delta / 2\right]$ (Fig. 4).

The resulting MAC protocol is simpler than $\mathrm{f}-\mathrm{MAC}$ as no coordination (careful determination of the $k_{i}$ ) among neighbouring nodes is needed. Bandwidth and delay bounds of the protocol are equal to $\mathrm{f}-\mathrm{MAC}$ if $T_{R S}=T_{\max }$ is selected. Basically, the collision-free behaviour of f-MAC is traded for simplicity of the described random MAC.

\footnotetext{
${ }^{2}$ It has to be noted that TDMA based protocols also have to deal with lossy media and are therefore unable to provide a $100 \%$ delivery probability.
} 
Table 2. Delivery probability $\mathrm{P}$ of the random transmission scheme.

\begin{tabular}{|c|c|c|c|}
\hline$N$ & $T_{\max }$ & $P_{S T}$ & $P_{B T}$ \\
\hline \hline 2 & $7 \delta$ & 0.651428 & 0.640000 \\
\hline 3 & $21 \delta$ & 0.944530 & 0.858846 \\
\hline 4 & $43 \delta$ & 0.984797 & 0.944530 \\
\hline 5 & $89 \delta$ & 0.998696 & 0.989094 \\
\hline 6 & $131 \delta$ & 0.999716 & 0.995770 \\
\hline 7 & $205 \delta$ & 0.999975 & 0.999134 \\
\hline 8 & $267 \delta$ & 0.999995 & 0.999667 \\
\hline
\end{tabular}

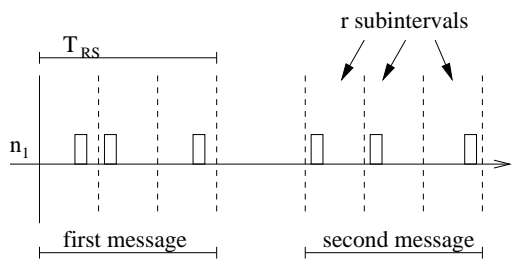

Fig. 4. Random transmission scheme.

\subsection{Traffic Analysis}

f-MAC attains a message delivery probability equal to 1 if it is assumed that only collisions will be responsible for packet losses. If $N$ nodes are contending for transmission, then $\mathrm{f}-\mathrm{MAC}$ requires each node to transmit $r=N$ framelets and every message will have been delivered after $T_{\max }$. With the random transmission scheme, a message is transmitted within the time $T_{R S}$ but the message could be lost due to collisions. Thus, the probability $P$ of a successful transmission is less than $1(P<1)$.

Saturated Traffic In this comparison it is assumed that $N$ nodes contending for the media are sending messages with the maximum available rate. If f-MAC is used in this traffic scenario, a message is sent every $T_{i}$ seconds, depending on the nodes specific $k_{i}$. If the random scheme is used, every node is sending a message every $T_{R S}$ seconds. To enable a fair comparison - both schemes provide the same bandwidth - the following value for $T_{R S}$ is selected: $T_{R S}=T_{\max }$. In this scenario, f-MAC attains a message delivery probability equal to 1 . For the analytic analysis it is assumed that all nodes start the transmission of messages at the same point in time. In this case, the random transmission scheme attains a transmission probability that can be calculated the following way:

$$
P\left(T_{R S}\right)=1-\left(1-\left(\frac{\frac{T_{R S}}{N}-\delta}{\frac{T_{R S}}{N}-\frac{\delta}{2}}\right)^{2 N}\right)^{N}
$$

The probability depends on the number of nodes that are in communication range of a potential message receiver. The delivery probability $P_{S T}=P\left(T_{R S}=\right.$ $\left.T_{\max }\right)$ is shown in Table 2 . For small $N$, the delivery probability is significantly lower than $100 \%$. In this operation area, the usage of the more complicated f-MAC sceme instead of the imsple random scheme is justified.

Bursty Traffic In this traffic scenario, it is assumed that sporadically an event triggers $N$ nodes to send a message to a receiver (similar to the application 
example described in Section 6). The time between events is assumed to be far larger than $T_{\max }$. Consequently, the channel will be temporarily saturated as nodes contend for transmission. Such a channel usage is for example likely if the nodes are sensor nodes. The sensor nodes in one area of the field might be triggered by the same event that all nodes are set-up to monitor.

If $\mathrm{f}-\mathrm{MAC}$ is used in this case, messages are guaranteed to be delivered after the time: $(r-1) \cdot t_{i}+\delta / 2$ (the waiting time $t^{\prime}$ must not be considered, as it is assumed that messages of previous events have been completely processed by the system). For comparison purposes, the same delivery time should be achieved by the random scheme. Thus, $T_{R S}=(r-1) \cdot t_{\max }+\delta / 2$ is selected.

The delivery probability $P_{B T}$ can be calculated using Equation (9). $P_{B T}=$ $P\left(T_{R S}=(r-1) \cdot t_{\max }+\delta / 2\right)$ is shown in Table 2 . Here again, the delivery probability for small $N$ is significantly lower than the $100 \%$ achieved by the f-MAC protocol.

\section{$4.3 \quad$ Findings}

In the real world, collision-free is not equal to $100 \%$ message delivery probability. Channel loss by interference will occur as well. However, in sparsely populated networks ( $\operatorname{small} N$ ), the probability of a message loss by collision can be considerable if a simple MAC protocol is used. f-MAC can reduce the number of message losses significantly as only message losses due to lossy links occur. Thus, f-MAC can be considered useful in sparsely populated networks with bursty traffic patterns if the link quality is not too bad.

\section{5 f-MAC Clusters}

The f-MAC scheme described previously allows only a collision-free delivery if all nodes participate in the scheme. Thus, if a large number of nodes have to communicate (e.g. many wireless sensors in a sensor field), the following problem arises. The number of framelets per transmission $r$ is equal to the number of nodes $N$ in the field. Additionally, $k_{\max }$ might be a high number so that Rule\#3 can be satisfied. This will result in a large $T_{\max }$ which finally defines the upper bound for transmission latency and the lower bound for bandwidth.

This problem can be attenuated by forming clusters. In most cases, not all nodes will be within the transmission range of all other nodes. Alternatively, the transmission power of the nodes can be dynamically adjusted such that only a few nodes are in transmission range of each other. Thus, the f-MAC scheme can be applied in localized areas that are formed by nodes that are within each others radio range.

\subsection{Cluster Example}

An example is depicted in Fig. 5. All nodes are assumed to have the same radio range $R$. Thus, $n_{1}, n_{2}$ and $n_{3}$ are within each others radio range and form cluster 
$C_{1}$. Within the cluster, the different $k_{i}$ have to be selected according Rule\#3. For example $k_{1}=3, k_{2}=5$ and $k_{3}=7$ can be used. Within cluster $C_{2}$, which comprises the next set of nodes that are in each others radio range, the $k_{i}$ have to be selected. As $n_{2}$ is member of 2 different clusters, it will use the already selected $k_{2}=5 . n_{5}$ and $n_{6}$ can not select the same $k_{i}$ used by $n_{1}$ and $n_{3}$ due to the hidden terminal problem. If for example $n_{5}$ would use $k_{5}=3$, messages sent by $n_{1}$ and $n_{5}$ to $n_{2}$ might collide at $n_{2}$. Thus, cluster $C_{1}$ and $C_{2}$ must obey the f-MAC rule set together. After computing the equations in Rule\#3, the following set of $k_{i}$ can be chosen for the clusters: $k_{1}=3, k_{2}=5, k_{3}=7$, $k_{4}=8, k_{5}=11$. Now, in $C_{1}$ and $C_{2} k_{\max }=11$ is obtained and the upper bound for the transmission delay is $T_{\max }=89 \cdot \delta$. Cluster $C_{3}$, containing another three nodes $\left(n_{5}, n_{6}, n_{7}\right)$ can now be added in a more simplistic way. $k_{5}=11$ can be maintained, for $n_{6} k_{6}=3$ can be selected as $n_{1}$ is not in the collision domain of $C_{3}$. For the same reason, $k_{7}=7$ can be selected for $n_{7}$. Now the clusters are setup and can be used with the f-MAC protocol.

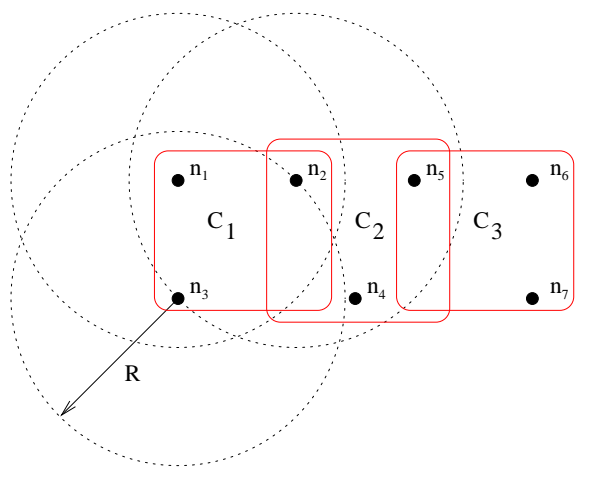

Fig. 5. f-MAC cluster forming.

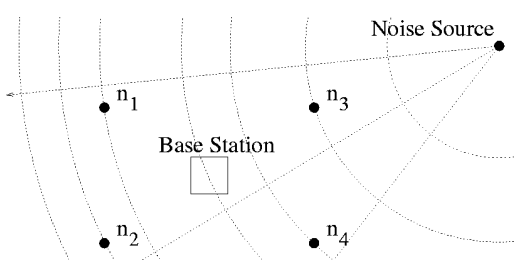

Fig. 6. Noise-event detection.

\subsection{Cluster Forming}

Obviously, a method is necessary to form clusters and compute the $k_{i}$ after a field is first set-up. This can be done statically after deployment, or a dynamic protocol for cluster forming has to be used. This paper focus on the investigation of the basic features of f-MAC and therefore cluster forming techniques are not discussed in this paper.

\section{6 f-MAC Application Areas}

The two exemplary application areas described in this Section benefit from the use of f-MAC. 
The first application is the use of f-MAC to realise deterministic sensor networks. As f-MAC provides hard guarantees on the information transfer delay, it can be used as one building block of a sensor network that can guarantee proper functioning in all possible operation cases. The second application is the use of f-MAC to realise a sensor network that is used for time critical event detection.

For both described areas other solutions than f-MAC exist. However, for some characteristics of the described applications, f-MAC represents the better solution.

\subsection{Worst Case Dimensioning}

Application areas for wireless sensor networks may encompass production surveillance, road traffic management, medical care or military applications. In these areas it is crucial to ensure that the sensor network is functioning even under worst case circumstances.

Analytical tools such as network calculus can be used to determine the numerical range of network properties such as message transfer delay and node buffer requirements [7]. It is then possible to dimension the sensor nodes in a way such that all possible (even the worst cases) traffic scenarios can be supported by the network. Supported means here that the specifications regarding message transfer delay or node buffer requirements stay within the defined bounds at any time.

To implement a sensor network that complies with the analytically determined specifications, deterministic network components are necessary. For example it is necessary to give a maximum upper bound for the message forwarding delay. Hence it is necessary to have a deterministic MAC layer. Contention based MAC protocols do not provide these guarantees. Collisions may occur and a back-off time is necessary. It is not possible to give an upper bound for the amount of back-offs that are necessary to transmit a message successfully. Thus, scheduled based MAC protocols are used if deterministic behaviour of the MAC protocol is required. These protocols have the desired deterministic behaviour but need a complicated and energy expensive time synchronisation among the nodes.

f-MAC provides the necessary deterministic behaviour in the MAC layer without costly time synchronisation. Thus, f-MAC can be seen as a building block for wireless sensor networks with deterministic behaviour.

\subsection{Time Critical Event Detection}

Sensor networks are often used to observe time critical events. For these kind of measurements it is necessary to associate measurements with the accurate point in time when they were taken. Particularly, if all measurements are analyzed at one point (e.g. a base station), it must be known at which point in time the measurements were taken. The example of such an application is shown in Fig. 6. The figure shows a base station and 4 sensors. The sensors are used to detect a specific acoustic event (e.g. a loud bang). Immediately after detection 
of the event a message is generated and sent to the base station. If the base station knows now when each of the sensors detected the event, it can calculate the position of the noise source. This is possible because the sound wave needs different times to reach each of the sensors.

Each sensor node places a time-stamp in each message sent towards the base station. Either a time synchronisation among the nodes is used or the base station determines the time offset of each node after the measurement was reported [6]. However, time synchronisation among nodes might not be possible or is too costly and the determination of time offsets after event detection needs additional protocol steps.

To work around these problems, f-MAC can be used. Each node detecting an event can immediately send a message to the base-station. The base station can then, knowing which framelet of a transmission was received and which $k_{i}$ was used, compute the time when the event was detected. This is possible as f-MAC provides a deterministic message delay. In this case no time synchronization among the nodes or additional protocol steps are necessary. Another advantage of using f-MAC for the described application is the fact that collisions can be handled. The detection of the acoustic event is very close together in time at each sensor. Therefore, if a contention based MAC protocol would be used, collisions would occur and the nodes would need to deal with this problem as well. This will increase the hardware complexity of the sensor node; an f-MAC sensor is simpler as it only has to send the framelets (no carrier sensing feature is necessary).

For the given application scenario, f-MAC provides a simpler alternative solution.

\section{Related Work}

MAC protocols for sensor networks can be coarsley classified in two groups: contention based and schedule based. Examples of contention based protocols are S-MAC [8] and T-MAC [9]. Representatives of the schedule based approach are TRAMA [10] and $\mu$-MAC [11]. The primary design goal of most MAC protocols for the area of wireless networks is energy efficiency and channel utilization. The primary design goal of the presented f-MAC protocol in contrast is a deterministic, collision-free behavior without time synchronization.

F-MAC shares with the contention based approach the lack of coordination between contending nodes for shared medium access. However, f-MAC differs from the cited contention based approaches because it is able to prevent message losses due to collisions with $100 \%$ guarantee. Schedule based protocols are are able to ensure collision-free communication but they invariably require some form of coordination between nodes. Mainly, time synchronisation among nodes is used which is not required in in the presented f-MAC protocol.

The Bitmac [12] protocol differs from the previously described, classical protocols and has similar properties and design goals as the presented f-MAC. Simmilar to f-MAC, collisions are not seen as "bad thing". If several nodes transmit a bit at the same time, the transmission collides and the receiver gets the bit- 
wise OR of the transmission. This feature can be used for specific applications in sensor networks. Additionally Bitmac has, similar to f-MAC, a deterministic behaviour. In difference to $\mathrm{f}-\mathrm{MAC}$, Bitmac requires synchronisation (time-division multiplexing) among nodes.

\section{Conclusion}

The paper presented the $\mathrm{f}-\mathrm{MAC}$ protocol that allows the implementation of a deterministic MAC layer without the need of time synchronization among the nodes. The protocol is collision-free and thus gives hard guarantees on message transfer delays and available bandwidth. The price for the deterministic behavior is a low channel utilization. However, some application scenarios might require a deterministic behavior and not necessarily a high throughput.

As shown, the message transfer delay increases exponentially with the number of nodes present in the same collision domain. Similarly, bandwidth degrades exponentially with the number of nodes in the same collision domain. Therefore, in most cases, f-MAC is only useful in sparsely populated networks.

The protocol is relatively simple to implement and therefore useful in areas where nodes are resource constrained. A timer is necessary within each node to implement the protocol. This timer is needed to time the transmission of the framelets and to measure when a next message can be transmitted. This timer has to be accurate only during the relatively short transmission time of one message. Thus, relatively simple clocks can be used which helps an implementation of $\mathrm{f}-\mathrm{MAC}$ in constrained environments.

As the delivery of at least one framelet of a message is guaranteed, a node does not have to perform a carrier sense. This is advantageous in a wireless environment as a reliable carrier sensing mechanism is difficult to implement. Additionally, the protocol deals with the hidden terminal problem. Two nodes which are not in radio range of each other can send simultaneously to a third node without additional protocol mechanisms (e.g. a CTS/RTS mechanism).

If power consumption has to be optimised within a network, f-MAC can be tuned in a way that duty cycles are implemented. In this case a message is repeated $j$ times by a node (a message is sent $j$ times using $r$ framelets for each message transmission). The receiver can then alter between a short energy intensive listen period and a long energy saving sleep period (sleeping $j-1$ times, awake 1 time). In this case, the message delay is increased by the factor $j$.

Clustering is necessary if f-MAC is used in a larger network. The cluster configuration might be obtained by a clustering protocol or via static configuration. Within a mobile environment, this might lead to an unacceptable communication overhead to maintain a cluster structure. Therefore, f-MAC might be only suitable for a non mobile network environment.

Consolidated, f-MAC is useful in constrained, static and sparsely populated networks where guarantees on the message transport delay are required.

The f-MAC protocol was implemented within a simulation environment and it was shown that the protocol has a low complexity and is therefore suitable in 
the sensor network environment. Currently we are incorporating the protocol in our sensor platform to evaluate the protocol in a real environment.

\section{Acknowledgments}

The support of the Informatics Research Initiative of Enterprise Ireland is gratefully acknowledged.

\section{References}

1. B. O'Flynn, A. Barroso, S. Bellis, J. Benson, U. Roedig, K. Delaney, J. Barton, C. Sreenan, and C. O'Mathuna. The Development of a Novel Miniaturized Modular Platform for Wireless Sensor Networks. In Proceedings of the IPSN Track on Sensor Platform, Tools and Design Methods for Networked Embedded Systems (IPSN2005/SPOTS2005), Los Angeles, USA, April 2005.

2. S. Mahlknecht and M. Boeck. CSMA-MPS: A Minimum Preamble Sampling MAC Protocol for Low Power Wireless Sensor Networks. In Proceedings of the 5th IEEE International Workshop on Factory Communication Systems (WFCS2004), Vienna, Austria, September 2004.

3. Nordic Inc. nRF2401 Single Chip 2.4GHz Radio Transceiver Data Sheet, 2002.

4. NannoNET. nanoNET TRX Transceiver Data Sheet, http://www.nanotron.com.

5. Chipcon. CC2420 Transceiver Data Sheet, http://www.chipcon.com, 2004.

6. G. Simon, M. Maroti, A. Ledeczi, G. Balogh, B. Kusy, A. Nadas, G. Pap, J. Sallai, K. Frampton: Sensor Network-Based Countersniper System, SenSys 04, Baltimore, USA, November 2004

7. J. Schmitt and U. Roedig. Sensor Network Calculus - A Framework for Worst Case Analysis. In Proceedings of the International Conference on Distributed Computing in Sensor Systems (DCOSS05), Marina del Rey, USA, June 2005.

8. W. Ye, J. Heidemann, and D. Estrin. An energy-efficient MAC protocol for wireless sensor networks. In Proceedings of the IEEE Infocom 2002 (INFOCOM2002), New York, USA, June 2002.

9. T. van Dam and K. Langendoen. An adaptive energy-efficient mac protocol for wireless sensor networks. In Proceedings of the First ACM Conference on Embedded Networked Sensor Systems (SenSys 2003), Los Angeles, USA, November 2003.

10. V. Rajendran, K. Obraczka, and J. Garcia-Luna-Aceves. Energy-efficient, collisionfree medium access control for wireless sensor networks. In Proceedings of the First ACM Conference on Embedded Networked Sensor Systems (SenSys 2003), Los Angeles, USA, November 2003.

11. A. Barroso, U. Roedig, and C. J. Sreenan. u-MAC: An Energy-Efficient Medium Access Control for Wireless Sensor Networks. In Proceedings of the 2nd IEEE European Workshop on Wireless Sensor Networks (EWSN2005), Istanbul, Turkey, January 2005.

12. M. Ringwald and K. Roemer. BitMAC: A Deterministic, Collision-Free, and Robust MAC Protocol for Sensor Networks. Proceedings of 2nd European Workshop on Wireless Sensor Networks (EWSN 2005), Istanbul, Turkey, January 2005.

13. B. Davidson and C. Bostian. A One-Way Packet Communication Channel with Retransmissions. SIGMOBILE Mobile Computing and Communication Review, vol. 2, no. 1, 1998. 